\title{
Sharp and fast: Sensors and switches based on polymer brushes with adsorption-active minority chains.
}

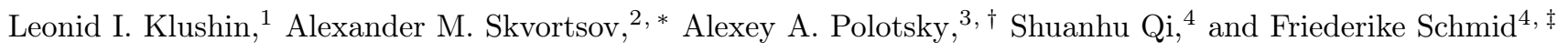 \\ ${ }^{1}$ Department of Physics, American University of Beirut, P.O. Box 11-0236, Beirut 1107 2020, Lebanon \\ ${ }^{2}$ Chemical-Pharmaceutical Academy, Prof. Popova 14, 197022 St. Petersburg, Russia \\ ${ }^{3}$ Institute of Macromolecular Compounds of Russian Academy of Sciences, \\ 31 Bolshoy pr., 199004 Saint-Petersburg, Russia \\ ${ }^{4}$ Institut für Physik, Johannes-Gutenberg Universität Mainz, Staudinger Weg 7-9, 55099 Mainz, Germany
}

\begin{abstract}
We propose a design for polymer-based sensors and switches with sharp switching transition and fast response time. The switching mechanism involves a radical change in the conformations of adsorption-active minority chains in a brush. Such transitions can be induced by a temperature change of only about ten degrees, and the characteristic time of the conformational change is less than a second. We present an analytical theory for these switches and support it by self-consistent field calculations and Brownian dynamics simulations.
\end{abstract}

Multicomponent polymer brushes offer promising perspectives for the design of smart responsive materials with a wide range of applications in nano- and biotechnology [1, 2]. For example, mixed polymer brushes comprising approximately equal amounts of hydrophobic and hydrophilic polymers have been used to fabricate surfaces with switchable wettability [3, 4. If the polymers phase separate along the direction perpendicular to the brush, the surface properties can switch between the properties of the two polymer species. The transition occurs on a temperature interval of $\Delta T \approx 30 \mathrm{~K}$. However, the response times are relatively slow on the time scale of minutes to hours, due to the existence of kinetically frozen metastable states with lateral nanoscale segregation.

In this letter we propose a new class of brush-based switches, which rely on a radical conformational change of adsorption-active minority chains in a brush. The basic mechanism of the transition is illustrated in Fig. 1 1 a). Consider a brush of polymers with chain length $N_{b}$ containing a small amount of minority chains with length $N>N_{b}$, which undergo an adsorption transition on the substrate. In the absence of the brush, the adsorption transition (in the limit $N \rightarrow \infty$ ) is continuous. Due to the interaction with the brush, it becomes first order at $N, N_{b} \rightarrow \infty$, and at finite chain length the chain end distribution of the minority chain becomes bimodal. A small change in temperature or solvent composition may thus lead to a sharp transition from an adsorbed state, where the switch chain is completely hidden inside the brush, to an exposed state, where the free end of the switch chain is localized at the outer surface of the brush. If each minority chain has an active group attached to its free end, the brush switches between two states: one where all active groups are fully hidden inside the brush, near the solid substrate, and one where they are exposed at the outer brush surface. The active end-groups can serve as sensors triggering an immune-like response or a detectable change in optical properties.

The proposed switches possess two main advantages.
First, the transition is sharp and can be induced by a temperature change of only about ten degrees, as attested and utilized by polymer chromatography in mixed eluents [5]. Second, the characteristic time scale for conformational changes is small; below, we estimate it to be well below a second. Hence the rate of change in brush properties is limited by the rate of change in the external conditions. We should note that a similar conformational transition can be observed when varying the contour length of the minority chain, and this has been studied in earlier work by theory [6] and simulations [7, 8]. However, this effect cannot be used for switching since it is almost impossible to change the contour length in a controlled and reversible way.

The purpose of the present letter is to provide a theoretical description for the proposed class of sensor switches and to clarify how the main brush parameters (the chain lengths $N_{b}$ and $N$, the grafting density $\sigma$ ) affect the two main characteristics of the switch performance: the sharpness of the transition from one state to the other, and the time of response to a sudden change in the control parameters. We develop an analytical theory and support it by self-consistent field (SCF) lattice calculations and Brownian dynamics simulations.

Evidence of the sharp transition. To illustrate the switch mechanism, we first present SCF results for model brushes of polymers with length $N_{b}=100$ containing a single adsorption-active chain of length $N$. The minority chain is subject to the SCF brush potential created by the other chains, plus an adsorption potential: Each monomer contact with the surface leads to an energy gain $\varepsilon$. In the following, all energies are given in units of the thermal energy, $k_{B} T$, and all lengths in units of the segment length, $a$. Fig. 1 $\mathrm{b}$ ) shows the average distance between the free end of the adsorption-active switch chain to the solid substrate, $\left\langle z_{e}\right\rangle$, as a function of $\varepsilon$ for brushes with grafting density $\sigma=0.2$. The curves are obtained by SCF calculations for walks on a 6 -choice simple cubic lattice. Already a small positive increment 
(a)

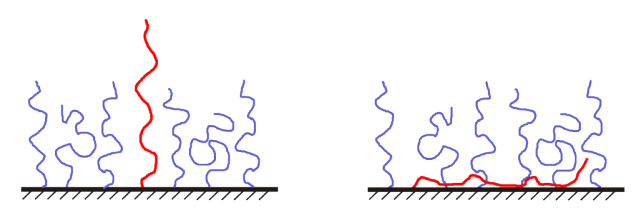

(b)

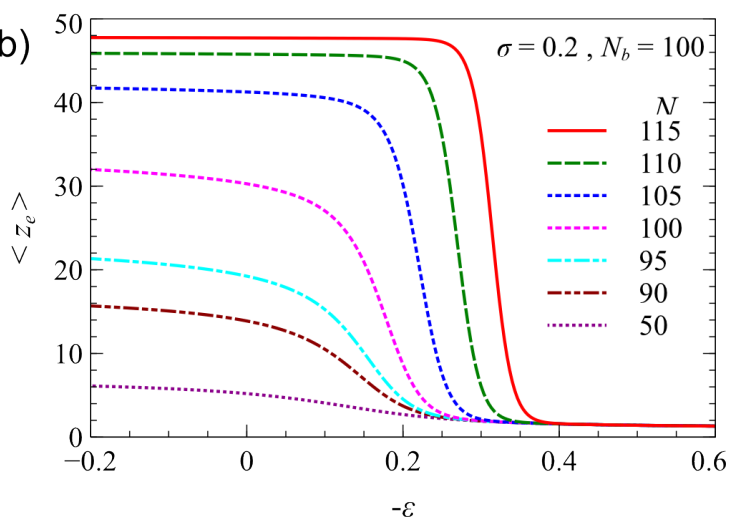

Figure 1. (a) Cartoon of the two states of the switching minority chain. (b) Average chain end height $\left\langle z_{e}\right\rangle$ vs. adsorption energy $\varepsilon$ for different values of the minority chain length $N$ (as indicated) in a brush with chain length $N_{b}=100$ and grafting density $\sigma=0.2$.

$\Delta=N-N_{b}$ in the length of the minority chain results in a pronounced transition from a stretched state in which the free end of the minority chain is localized at the outer surface of the brush to an adsorbed state with the switch chain localized very close to the substrate. For negative or zero increment, the transition is considerably smoother, and the desorbed state displays large fluctuations of the chain end position, as typical for polymer brushes [9]. In contrast, the end fluctuations for desorbed chains with positive chain length increment $\Delta$ are much smaller, and the peak of the distribution is located near the brush edge [6, 8]. This is illustrated in Fig. 2a), which shows the logarithm of the end distribution (the Landau free energy for chains with end position $z$ ) for different switch chain length $N$ at the mid-point of the transition, i.e., the adsorption strength $\varepsilon_{m}$ where the switch chain can be found in the adsorbed and the desorbed state with equal probability. The free energy has two distinct minima, hence the two states are clearly separated. The location of the minimum at large $z$ corresponds exactly to the most probable chain end position in the absence of an attractive surface potential. The minimum at small $z$ refers to rather strongly adsorbed chains since the adsorption energies are considerably larger than the critical adsorption energy $\varepsilon_{c}=-\ln (6 / 5) \simeq-0.182$ [10]. With increasing $N$, the minima become separated more strongly and the transition sharpens, i.e., the width of the transition in $\varepsilon$ decreases (Fig. 2b).

Theory. The theoretical description of the two competing states of the adsorption-active minority chain of length $N$ surrounded by the non-adsorbing chains of length $N_{b}$ is based on several simplifying assumptions:
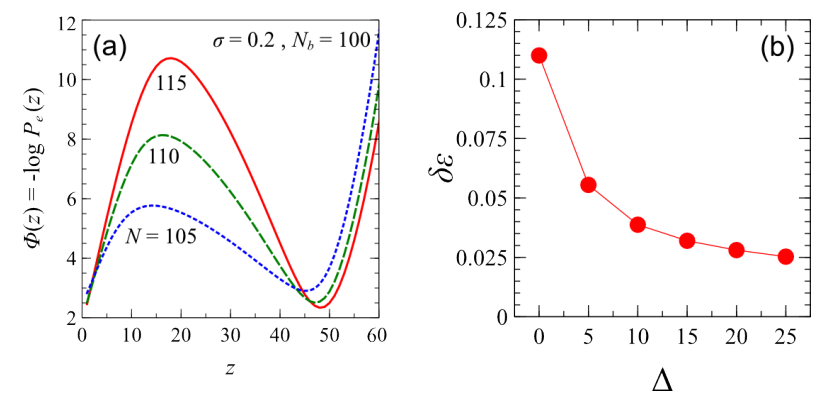

Figure 2. (a) Landau free energy (logarithm of the end distribution $\left.P_{e}(z)\right)$ for switch chains of different length $N$ integrated into a brush with chain length $N_{b}=100$ and grafting density $\sigma=0.2$, at the mid-point $\varepsilon_{m}$ of the transition. Solid red line: $N=105, \varepsilon_{m}=0.190$; dashed green line: $N=110, \varepsilon_{m}=0.246$; dotted blue line: $N=115, \varepsilon_{m}=0.293$. (b) Width of the transition between the adsorbed and desorbed chain, defined as $\delta \varepsilon=H \cdot\left(\mathrm{d}\left\langle z_{e}\right\rangle / \mathrm{d} \varepsilon\right)^{-1}$ evaluated at $\varepsilon_{m}$ ( $H$ is the brush height) as a function of chain increment $\Delta=N-N_{b}$.

First, we treat the inter- and intrachain interactions in the mean-field approximation. Second, we assume that minority chains are grafted far enough from each other so that their mutual interaction is negligible compared to interactions of the minority chains with the surrounding brush chains. Third, we neglect the effect of the change in the minority chain conformation on the surrounding brush. Hence, the conformation of a minority chain is determined by a fixed mean-field potential profile consisting of the repulsive contribution determined by the brush density and a short-ranged attraction to the solid substrate. The minority chain itself is described by an ideal continuum model, since intrachain excluded volume effects are screened out considerably within the brush thickness. The Green's function $G(z, N)$, i.e. the total statistical weight of the minority chain grafted at the substrate $\left(z_{0}=0\right)$ as a function of the free end position, $z$, is a solution of the Edwards equation

$$
\frac{\partial G(z, s)}{\partial s}=\frac{1}{6} \frac{\partial^{2} G(z, s)}{\partial^{2} z}-V(z) G(z, s),
$$

where $s$ is the number of monomers. The total potential is $V(z)=V_{b}(z)+V_{a d s}(z)$, where the mean field due to a brush of thickness $H$ is known from analytical theory [11:

$$
V_{b}(z)=\frac{3 \pi^{2}}{8 N_{b}^{2}}\left(H^{2}-z^{2}\right),
$$

and the adsorption interaction is described by an attractive pseudopotential of strength $c, V_{a d s}(z)=-c \delta(z)$. Close to the substrate, $z \ll H$, the brush potential is roughly constant, $V_{b}(z) \simeq \frac{3 \pi^{2}}{8 N_{b}^{2}} H^{2} \equiv U_{0}$. For moderate grafting densities, $\sigma \lesssim 0.25$, the analytical theory gives a simple relation, $U_{0}=\frac{3}{2}\left(\frac{\pi \sigma}{2}\right)^{2 / 3}[11$. 
The adsorbed state is thus approximately described by the standard partition function modified by the potential $U_{0}$ [12]:

$$
Q_{a d s}=2 e^{N\left(-U_{0}+c^{2} / 6\right)}
$$

The state with the free end exposed at the brush edge has no contacts with the surface. Hence, the Green's function is given by the known solution of the Edwards equation for a purely parabolic potential and a neutral solid surface [6] :

$$
G_{e x}(z, N)=\frac{\pi}{2}\left(\frac{3}{N_{b} \sin \left(\frac{\pi N}{2 N_{b}}\right)}\right)^{3 / 2} z e^{-\frac{3 \pi}{4 N_{b}} \cot \left(\frac{\pi N}{2 N_{b}}\right) z^{2}},
$$

For minority chains with positive increment, $N>N_{b}$, the Green's function, Eq. (4), increases monotonically with $z$ and is unbounded, since it refers to a parabolic potential (2) extending to infinity. In reality, the potential is cut off at $z=H$, and the minority chain does not make excursions well beyond $z=H$. To account for this, we truncate $G_{e x}(z, N)$ at $z=H$. If minority chains are just slightly longer $\Delta \equiv N-N_{b} \ll N_{b}$, (which is of prime interest for this letter) the Green's function simplifies to:

$$
G_{e x}(z, N)=\left\{\begin{array}{cl}
\frac{\pi}{2}\left(\frac{3}{N_{b}}\right)^{3 / 2} z e^{\left(U_{0} \Delta\right)\left(\frac{z}{H}\right)^{2}} & , z \leq H \\
0 & , z>H
\end{array}\right.
$$

which gives the partition function

$$
Q_{e x}=\frac{2}{\pi \Delta}\left(3 N_{b}\right)^{1 / 2} e^{U_{0} \Delta}
$$

assuming $e^{U_{0} \Delta} \gg 1$. Eqs. (3), (6) clearly show that in the limit of $N_{b} \rightarrow \infty, \sigma=$ const, $\frac{\Delta}{N_{b}}=$ const, the free energies of the two states are extensive and switching becomes a classical first-order phase transition. In the desorbed state, the chain end position $z_{e}$ takes the mean value $\left\langle z_{e}\right\rangle \gtrsim H \propto N_{b}$ and the amplitude of fluctuations roughly scales like $\Sigma_{e}=\sqrt{\left\langle z_{e}^{2}\right\rangle-\left\langle z_{e}\right\rangle^{2}} \propto \sqrt{\Delta}$. In the adsorbed state, $\left\langle z_{e}\right\rangle$ and $\Sigma_{e}$ are of the same order (the adsorption blob size) and close to zero. Hence the two states are well separated.

The theory can be used to derive approximate expressions for the transition point and other quantities of interest. The transition point is defined by the condition $Q_{a d s}\left(c^{*}\right)=Q_{e x}$. Omitting logarithmic corrections this gives $c^{*}=\left(6 U_{0} \Delta / N\right)^{1 / 2}$. The connection between the pseudopotential strength $c$ and the lattice parameter $\varepsilon$ for 6-choice walks is known [13] and can be linearized close to the critical adsorption point as $c=5\left(\varepsilon_{c}-\varepsilon\right)$. Thus the prediction for the transition point in the lattice model reads

$$
\left(\varepsilon_{c}-\varepsilon^{*}\right)=\frac{3}{5}\left(\frac{\pi \sigma}{2}\right)^{1 / 3}\left(\frac{\Delta}{N}\right)^{1 / 2}
$$
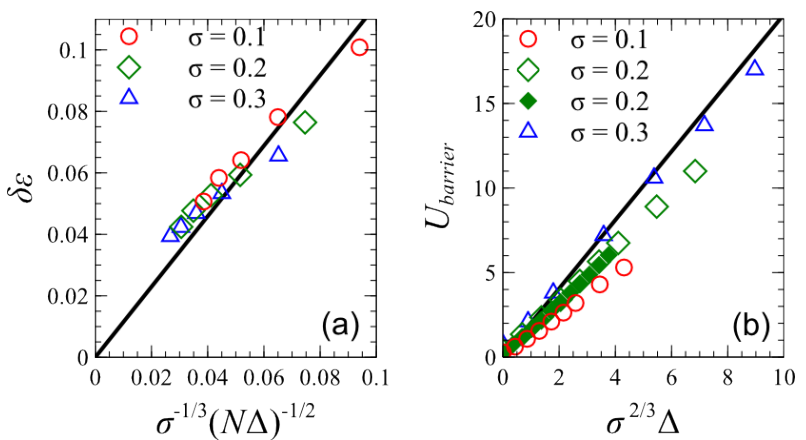

Figure 3. Scaling plots for the width of the transition (a) and the barrier height for the adsorbing minority chain (b) in brushes with different grafting densities, plotted according to the theoretical predictions of of Eqs. (8) (a) and (9). Here $N$ is the minority chain length and $\Delta=N-N_{b}$ the chain length difference between switch chains and brush chains $\left(N_{b}\right.$ is the brush chain length). Open symbols represent SCF data for $N_{b}=100$ and $\sigma=0.1,0.2,0.3$; filled diamonds in panel (b) show data from single-chain Langevin dynamics simulations, see text below.

The sharpness of the transition, characterized by the inverse slope $\delta \varepsilon$ of $\left\langle z_{e}\right\rangle$ at the mid-point of the transition (see Fig. 2b), can be estimated from a standard recipe for the two-state model [14] $\delta \varepsilon=4 /\left(\mathrm{d} \ln Q_{a d s} / \mathrm{d} \varepsilon\right)_{\varepsilon=\varepsilon *}$, which combined with Eq. (3) results in

$$
\delta \varepsilon=\frac{4}{3}\left(\frac{\pi \sigma}{2}\right)^{-1 / 3}(N \Delta)^{-1 / 2} .
$$

The SCF data are in accordance with this analytical scaling prediction, see Fig. 3 a).

Finally, we consider the height of the barrier separating the adsorbed and exposed state, which is one important determinant of the switching time. The sawtooth shape of the free energy potential $\Phi(z)$ in Fig. 22 ) suggests that the transition states mostly correspond to squeezed desorbed chains. Hence we estimate the barrier height at the transition point, $U_{\text {barrier }}$, by the change in the Green's function of the exposed state, $U_{\text {barrier }}=\ln \frac{G_{e x}(z=H)}{G_{e x}\left(z=z_{\text {barrier }}\right)}$. From Eq.(5), it follows that up to a numerical coefficient close to 1 ,

$$
U_{\text {barrier }} \simeq U_{0} \Delta=\frac{3}{2}\left(\frac{\pi \sigma}{2}\right)^{2 / 3} \Delta .
$$

Fig. 3p) shows that the SCF data again agree reasonably well with the analytical scaling prediction at sufficiently high grafting density. Here the numerical data were obtained for the adsorption strength where the two minima of $\Phi(z)$ have equal depth (which is slightly different from the mid-point $\left.\varepsilon_{m}\right)$.

Switching times. The expression for the barrier height, Eq. 9. can be used to derive a simple estimate for the switching time 15

$$
\tau_{\text {switch }}=2 \pi \tau_{0} \exp \left(U_{\text {barrier }}\right)
$$




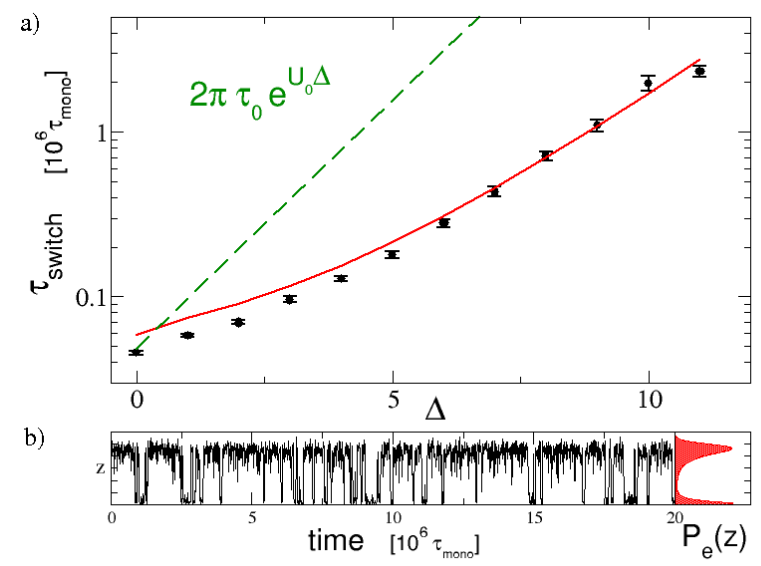

Figure 4. (a) Mean switching times for the switch chain in a brush with brush chain length $N_{b}=100$ and grafting density $\sigma=0.2$ at the adsorption transition (see text) as a function of the length increment $\Delta=N-N_{b}$. Symbols: data from Brownian dynamics simulations; dashed green line: Arrhenius estimate (Eq. 10p); solid red line: refined estimate based on Kramers' theory (Eq. (11)) with $N_{\text {eff }}=N-N_{b} / 3$. (b) Time evolution of the end monomer position $z$ and corresponding end monomer distribution $P_{e}(z)$ for $\Delta=6$.

where the time scale $\tau_{0}$ is related to the rate at which the chain attempts to overcome the barrier. A robust estimate from above is obtained by taking the relaxation time in the absence of the barrier, i.e., the relaxation time for switch chains with length $N=N_{b}$ at $\varepsilon=0$. The single-chain relaxation time in the presence of the mean field created by the surrounding brush scales as $\tau_{0} \sim N_{b}^{3} \sigma^{2 / 3} \tau_{\text {mono }}$ [16, 17] where $\tau_{\text {mono }}$ is the characteristic monomer relaxation time which is typically on the order of $10^{-9} s$ for flexible chains.

In order to obtain a more realistic description of the switching dynamics, we have carried out overdamped Brownian dynamics simulations of Gaussian switch chains in the same SCF brush potential used for the previous calculations (at $N_{b}=100, \sigma=0.2$ ), plus an exponentially decaying adsorption potential with adsorption strength chosen such that the end monomer distribution, $P_{e}(z)$, has equal height in the adsorbed and desorbed state. The static properties of the Gaussian chains are almost identical to those of the corresponding lattice chains. For example, the barrier height derived from the chain end distribution $P_{e}(z)$ distribution is the same (see Fig. 3b). The mean switching time between states (averaged over both switching directions) is shown in Fig. 4 a) as a function of the chain length increment $\Delta$ and compared with Eq. 10 . Here the singlechain relaxation time in the brush, $\tau_{0}$, was determined from independent simulations at $\Delta=0$ and $\varepsilon=0$ (giving $\tau_{0}=7,7 \cdot 10^{3} \tau_{\text {mono }}$ ). Eq. 10 strongly overrates the switching time already for small increments $\Delta$, and the deviations become worse for larger $\Delta$. A better estimate is obtained if one accounts for the full shape of the free energy profile according to Kramers' theory [15]

$$
\tau_{\text {switch }}=\frac{1}{2} N_{\text {eff }} \tau_{\text {mono }} \int_{z_{a d s}}^{z_{e x}} \mathrm{~d} z \int_{0}^{\infty} \mathrm{d} z^{\prime} e^{\Phi(z)-\Phi\left(z^{\prime}\right)},
$$

where $z_{a d s}$ and $z_{e x}$ are the positions of the two minima of $\Phi(z)$. Here $N_{\text {eff }}$ is an adjustable parameter denoting the effective number of monomers that participate in the switching process. $N_{\text {eff }}$ is smaller than $N$ due to the fact that the monomers close to the grafted end remain close to the surface even in the exposed state. The simulation data can be fitted reasonably well with $N_{\text {eff }} \approx N-N_{b} / 3$ (Fig. 4).

According to the simulation results shown in Fig. 4 . a switch based on the proposed mechanism could have switch times in the range of $10^{6} \tau_{\text {mono }}$, i.e., milliseconds. In real brushes, the coupling of the minority chain with the brush will slow down the relaxation times [18. On the other hand, hydrodynamic interactions, which have also been neglected in the present work, tend to facilitate cooperative motions and will likely accelerate the switching dynamics.

In sum, we have presented a mechanism that could be used to design smart switchable surfaces with very short response times. The switching times are much faster than in conventional stimulus-responsive surfaces based on mixed brushes, because they do not involve a cooperative reorganization of an entire polymer layer. The theory developed above indicates that the two main requirements for an optimal switching process, i.e., fast response times and high sensitivity to changes in the environment, are at conflict with each other. The width of the transition decreases with increasing chain length increment $\Delta$ of the switch chain (Fig. 2b)), which increases the sensitivity, but at the same time, the switching time increases (Fig. 4a). Nevertheless, we can identify a regime, $\Delta / N_{b} \sim 10 \%$, where the transition is sharp while the switching times are still well in the subsecond regime.

The mechanism relies on the fabrication of dense brushes in the strongly stretched limit. Such brushes are typically not monodisperse. Nevertheless, the switching mechanism should still be effective. Polydisperse brushes also create a potential barrier between the adsorbed and desorbed state of the switch chain. Chain end fluctuations of brush chains are reduced in polydisperse brushes [19, 20], hence the switching transition might even become sharper in the regime of small $\Delta$ where the lengths of switch chain and brush chains are comparable.

\section{Acknowledgements}

Financial support by the Deutsche Forschungsgemeinschaft (Grant SCHM 985/13-1, and 13-03-91331-NNIOa) is gratefully acknowledged. We thank K. Binder for helpful discussions. Simulations have been carried out on the compute cluster Mogon at JGU Mainz. 
* astarling@yandex.ru

† alexey.polotsky@gmail.com

‡ friederike.schmid@uni-mainz.de

[1] M. Cohen Stuart, W. Huck, J. Genzer, M. Muller, C. Ober, M. Stamm, G. Sukhorukov, I. Szleifer, V. Tsukruk, M. Urban, F. Winnik, S. Zauscher, I. Luzinov, and S. Minko, Nature Materials 9, 101 (2010)

[2] T. Chen, R. Ferris, J. Zhang, R. Ducker, and S. Zauscher, Progress in Polymer Science 35, 94 (2010)

[3] J. Draper, I. Luzinov, S. Minko, I. Tokarev, and M. Stamm, Langmuir 20, 4064 (2004)

[4] M. Motornov, S. Minko, K. Eichhorn, M. Nitschke, F. Simon, and M. Stamm, Langmuir 19, 8077 (2003)

[5] A. Abdulahad and C. Ryu, J.Pol.Sci. B-Polymer Physics 47, 2533 (2009)

[6] A. Skvortsov, L. Klushin, and A. Gorbunov, Macromolecules 30, 1818 (1997)

[7] D. Romeis, H. Merlitz, and J. Sommer, J.Chem.Phys. 137, 064907 (2012)

[8] D. Romeis and J. Sommer, J.Chem Phys 139, 044910 (2013)
[9] P. I. Lai and E. B. Zhulina, Journal de Physique II 2, 547 (1992)

[10] R. Rubin, J. Chem. Phys. 43, 2392 (1965)

[11] E. Zhulina, O. Borisov, and V. Priamitsyn, J.Coll. Interface Sci. 137, 495 (1990)

[12] A. Skvortsov, A. Gorbunov, F. Leermakers, and G. J. Fleer, Macromolecules 32, 2004 (1999)

[13] A. Gorbunov, A. Skvortsov, J. van Male, and G. Fleer, J. Chem. Phys. 114, 5366 (2001)

[14] M. Challa, D. Landau, and K. Binder, Phase Transitions: A Multinational Journal de Physique II 24-26, 43 (1990)

[15] W. Paul and J. Baschnagel, Stochastic Processes: From Physics to Finance (Springer, Berlin, 1999)

[16] L. Klushin and A. Skvortsov, Macromolecules 24, 1549 (1991)

[17] M. Murat and G. Grest, Macromolecules 22, 4054 (1989)

[18] D. Reith, A. Milchev, P. Virnau, and K. Binder, Macromolecules 95, 28003 (2011)

[19] L. Klushin and A. Skvortsov, Macromolecules 25, 443 (1992)

[20] W. M. de Vos and F. A. M. Leermakers, Polymer 50, 305 (2009) 